\title{
Shape-dependent plasmon resonances of gold nanoparticles
}

\author{
Colleen L. Nehl $\dagger$ and Jason H. Hafner* \\ Received 28th September 2007, Accepted 7th January 2008 \\ First published as an Advance Article on the web 11th February 2008 \\ DOI: $10.1039 / b 714950 f$
}

Localized surface plasmon resonances in noble metal nanoparticles cause enhanced optical absorption and scattering that is tunable through the visible and near-infrared. Furthermore, these resonances create large local electric field enhancements at the nanoparticle surfaces, essentially focussing light at the nanometer scale. These properties suggest a range of applications, including biomedical imaging, therapeutics, and molecular sensing. Here we review some recent advances regarding shape-dependent optical properties of two specific nanoparticle geometries: gold nanorods and branched gold nanoparticles.

\section{Introduction}

The past decade has seen significant advances in the synthesis and fabrication of monodisperse noble metal nanoparticles with a variety of shapes, from simple spheres to branched multipods. These particles are pursued for applications in nanophotonics due to an effect called localized surface plasmon resonance (LSPR). LSPR occurs when an electromagnetic field drives the collective oscillations of a nanoparticle's free electrons into resonance. This classical effect was theoretically described by Mie in 1908 by solving Maxwell's equations for a plane wave incident on a metal sphere surrounded by a dielectric medium, given the dielectric function of the metal. Mie theory provides an exact analytical solution for nanosphere LSPR in terms of a multipole expansion. For nanospheres whose diameters are less than about one tenth the incident radiation wavelength, only the dipole term in the expansion is significant, and the extinction simplifies to:

Department of Physics \& Astronomy, Rice University, 6100 Main Street, Houston, TX, USA. E-mail: hafner@rice.edu; Fax: +1 713348 4150, Tel: +17133483205

$\dagger$ Current address: Department of Chemistry, Northwestern University, 2145 Sheridan Road, Evanston, IL, USA. E-mail: c-nehl@ northwestern.edu

$$
\sigma=\frac{18 \pi V \varepsilon_{\mathrm{m}}{ }^{3 / 2}}{\lambda} \frac{\varepsilon_{2}}{\left(\varepsilon_{1}+2 \varepsilon_{\mathrm{m}}\right)^{2}+\varepsilon_{2}{ }^{2}}
$$

where $\sigma$ is the extinction cross section, $V$ is the nanoparticle volume, $\lambda$ is wavelength, $\varepsilon_{\mathrm{m}}$ is the dielectric constant of the medium, and $\varepsilon_{1}+\mathrm{i} \varepsilon_{2}$ is the complex dielectric function of the metal. ${ }^{1}$ The resonance condition is met when $\varepsilon_{1}=-2 \varepsilon_{\mathrm{m}}$, which occurs in the visible for gold and silver nanospheres. The sharpness of the resonance is determined by the imaginary part of the dielectric function, $\varepsilon_{2}$. Silver, having a smaller imaginary component than gold, exhibits sharper, brighter resonances. ${ }^{2}$

One can see that in the dipole approximation, LSPR has no dependence on the size of the nanosphere. The resonant condition is purely determined by the dielectric functions of the metal and the medium. However, for larger nanospheres where the higher multipoles become important, there is a modest red shift with size. For example, $20 \mathrm{~nm}$ diameter gold nanoparticles have a LSPR at a wavelength of $520 \mathrm{~nm}$ in water, which red shifts to $600 \mathrm{~nm}$ as the nanoparticle diameter increases to $100 \mathrm{~nm}^{1}$ Beyond this size, the resonance continues to shift but becomes significantly broadened. This broadening is due to both the different peak wavelengths of the higher multipoles and the radiative damping of the resonance as the scattering cross section rapidly increases.

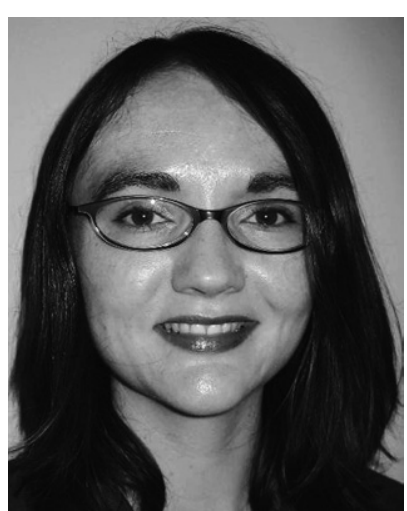

Colleen Nehl
Dr Colleen Nehl earned her Ph.D. in physics from Rice University in 2007. She is currently a postdoctoral research associate at Northwestern University in Professor Teri Odom's laboratory where she studies the fabrication and optical properties of novel plasmonic nanostructures created by phase-shift photolithography.

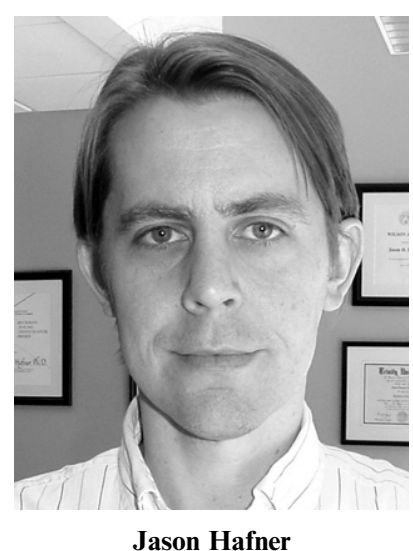

Dr Jason Hafner is an Assistant Professor at Rice University in the Department of Physics \& Astronomy. His group studies plasmon resonances in gold nanoparticles and their biological and biomedical applications. His research interests also include electrostatic analysis of lipid membranes by atomic force microscopy. 
The key to achieving broad LSPR tunability without sacrificing the linewidth of the resonance is to vary the nanoparticle shape. This Application article will describe how nanoparticle shape and size affect LSPR properties. The wide array of nanoparticles and applications pursued to date are well beyond the scope of this review. We will therefore focus on recent advances in two types of chemically synthesized nanoparticles: simple gold nanorods and more complicated branched gold nanoparticles. Other shapes can be synthesized in high yield, including gold nanoshells, ${ }^{3}$ nanocages, ${ }^{4}$ and various polyhedra. ${ }^{5,6}$ Other fabrication routes to LSPR nanoparticles include high resolution lithography, metal deposition in hard templates, and even hybrid synthesis/fabrication techniques, but these will not be reviewed here.

\section{Gold nanorods}

Gold nanorods like those displayed in Fig. 1 are perhaps the most widely studied tunable plasmon resonant nanoparticles. ${ }^{7}$ These cylindrical nanostructures, typically $10-20 \mathrm{~nm}$ in diameter and 10's to 100's of nanometers in length, were first synthesized by a surfactant-directed electrochemical method, ${ }^{8}$ and later by a simple electroless deposition of gold ions onto seed particles in the presence of a surfactant. ${ }^{9,10}$ Modifications of this synthesis produced nanorods in high yield. ${ }^{7,11}$ This seed-mediated, surfactant-directed synthesis is quite powerful and can produce a variety of other shapes. ${ }^{12}$ Given the advanced state of their production, most recent experimental work on gold nanorods has focused on further manipulation of their surface chemistry, ${ }^{13-24}$ formation of complex assemblies, ${ }^{25-33}$ and development of biomedical and sensing applications. ${ }^{34-56}$

Gold nanorod extinction spectra exhibit two plasmon bands which correspond to LSPR along their length (longitudinal resonance) and across their diameter (transverse resonance). ${ }^{57}$ Although the lack of spherical symmetry precludes an exact analytical description of their LSPR by Mie theory, gold nanorod spectra are qualitatively described by Gans theory, which is an extension of Mie theory for spheroidal nanoparticles. Gans theory calculates a depolarization factor along each axis of the spheroid based on the aspect ratio of the semimajor and semiminor radii. In both Gans theory for spheroids and experimental

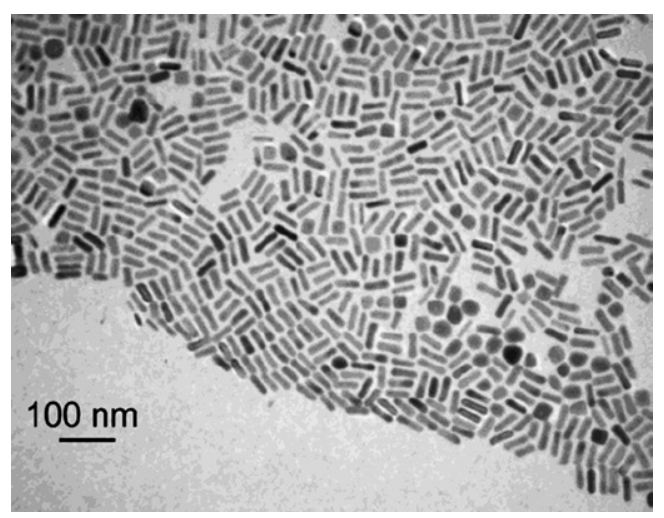

Fig. 1 An electron micrograph of monodisperse gold nanorods synthesized by seed-mediated, surfactant-directed synthesis. Adapted from reference 7 . measurements on gold nanorods, as the aspect ratio increases, the longitudinal mode along the semimajor axis red shifts and the transverse resonance along the semiminor axis slightly blue shifts. However, the agreement is only qualitative. To quantitatively fit a nanorod spectrum with Gans theory usually requires an unrealistically large dielectric constant for the medium..$^{56,57}$ Also, Gans theory overestimates the size of the extinction cross section relative to recent experimental measurements. ${ }^{58,59}$

Note that Gans theory only considers the dipole term of Mie theory, so it is only applicable to nanorods with lengths lower than $c a .80 \mathrm{~nm}$. The problem of higher order multipoles in gold nanorods has received less attention, in part since the high yield synthesis methods produce nanorods in the dipolelimited size range. However, template and other approaches can create larger nanorods ${ }^{60}$ and the higher order modes can be observed. ${ }^{61}$ For example, the higher order resonances of gold nanorods have been numerically calculated by the discrete dipole approximation (DDA) ${ }^{61,62}$ which represents a nanoparticle as a cubic array of polarizable points and calculates extinction cross sections based on the induced dipoles. Although DDA does not include higher order multipoles explicitly, they come about due to interactions of the dipoles. The T-matrix formulation, which directly calculates high order multipole effects, has also been applied to gold nanorod extinction..$^{63}$ Both DDA and $\mathrm{T}$-matrix predict the higher order multipole resonances for large gold nanorods.

An accurate description of gold nanorod LSPR must also carefully account for the structure of the endcap. While the early lower-yield synthesis methods usually produced hemispherical endcaps, the newer high yield methods often produce flat endcaps, or even flared ends that create "dog bone" shapes. As seen in Fig. 2, the effect of the endcap shape can significantly impact the LSPR resonance wavelength, causing up to $100 \mathrm{~nm}$ shifts from where one would expect the resonance based on the aspect ratio according to DDA calculations. ${ }^{64}$ The end shape effects were also considered in recent T-matrix calculations. ${ }^{63}$

\section{Branched gold nanoparticles}

In addition to elegant geometries such as rods, shells, and polyhedra, the LSPR of nanoparticles with more intricate shapes

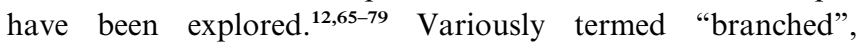
"multi-pod", "star-shaped", "lumpy", and even "sea urchinlike", these nanoparticles are synthesized by reduction of gold ions in a similar manner to other colloids. Several examples of branched gold nanoparticles are displayed in Fig. 3. They are generally not as highly monodisperse as other shapes, but in some cases well defined peaks in the ensemble spectra can be observed. Hao et al. ${ }^{66}$ found that three-tipped nanoparticles (the most prevalent shape in their sample) exhibited plasmon resonances at $700 \mathrm{~nm}$ and $540 \mathrm{~nm}$. DDA calculations on a model structure demonstrated that the $700 \mathrm{~nm}$ feature corresponded to a dipole resonance in the plane of the nanoparticle, and the 540 $\mathrm{nm}$ feature corresponded to a quadrupole resonance out of the plane of the nanoparticle.

With varying tip geometries and asymmetric shapes, one might expect branched nanoparticles to have complicated LSPR spectral features that are lost in ensemble measurements. To test this hypothesis, scattering spectra were measured for 

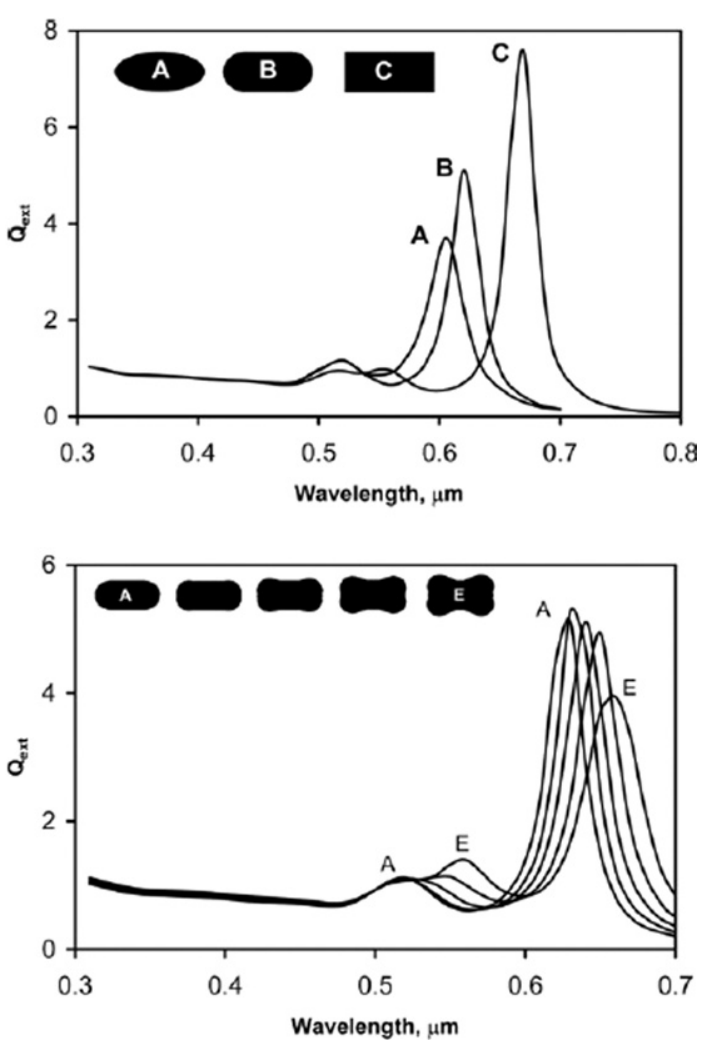

Fig. 2 The dependence of nanorod LSPR spectra on endcap shape, for several shapes which can result from high-yield gold nanorod synthesis. Adapted from reference 64 .

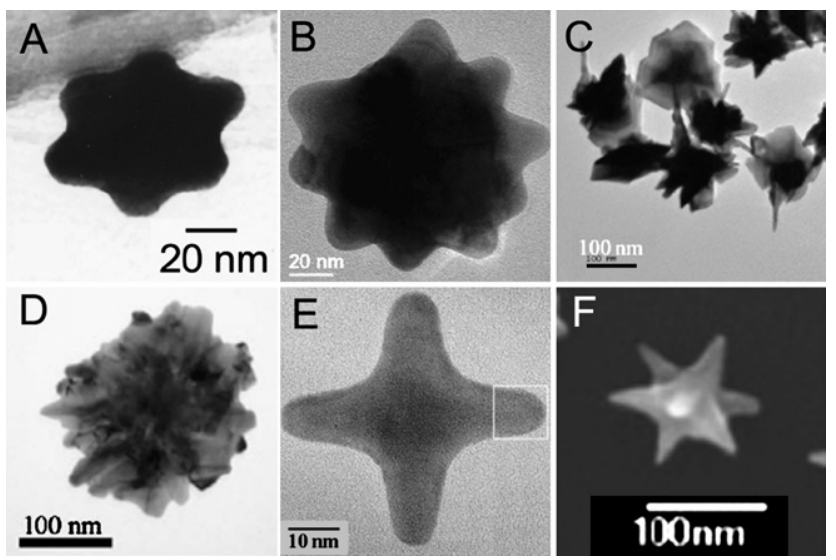

Fig. 3 Several different shapes of branched gold nanoparticles from references 67 (A), 69 (B), 75 (C), 77 (D), 65 (E), 72 (F).

individual gold "nanostars" by single particle spectroscopy. ${ }^{72}$ With the aid of alignment marks, high resolution electron micrographs of the individual nanostars were correlated with their scattering spectra. Typical single particle spectra consisted of multiple sharp peaks in the visible and near-infrared, as seen in Fig. 4. To find if the spectral peaks corresponded to a specific tip, an analyzer was placed on the scattered light. By tracking the peak amplitudes as a function of analyzer angle, it was found that each spectral peak from a single nanostar was polarized in a different direction, and each such direction matched that of a tip on the star.
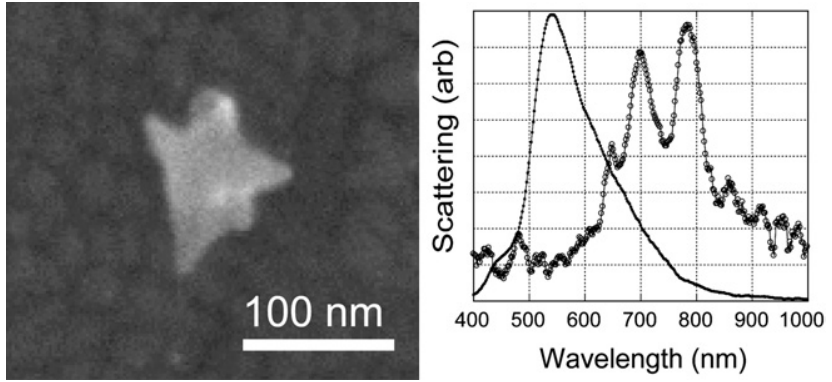

Fig. 4 The structure and scattering spectrum of a single gold nanostar. The smooth spectrum is that of a $100 \mathrm{~nm}$ nanosphere shown for reference. Adapted from reference 72 .

Further evidence of the correlation between the structure and spectra of gold nanostars was provided through numerical simulation of nanostar optical properties by FDTD (finite difference time-domain) analysis. ${ }^{74,79}$ FDTD is a brute force method which solves Maxwell's equations repeatedly along a time-evolving spatial grid, yielding information about the far-field extinction as well as the near-field enhancements at the nanoparticle surface. In one case, ${ }^{79}$ FDTD simulations were carried out on a structure modeled after an individual nanostar with a measured spectrum. ${ }^{72}$ The spectral extinction determined by FDTD was in excellent agreement with the experimental observations. In addition, analysis of the near-field enhancements confirmed that the observed resonances are localized about a single tip on the nanostar. ${ }^{74,79}$ In both reports, field enhancements of $c a$. 100 to 250 were found at the nanostar tips. These values are similar to those calculated for nanoparticle dimer junctions, ${ }^{80}$ yet exist in an open geometry, suggesting that the nanostars could be the basis for efficient SERS substrates.

LSPR in complex structures such as these branched gold nanoparticles can be understood in terms of the plasmon hybridization $(\mathrm{PH})$ model. $^{81} \mathrm{PH}$ is an analytical method which can calculate the LSPR of complicated structures by considering them to be the result of the interactions of the LSPR of simpler structures. Although PH has been successful for several geometries, ${ }^{82}$ a branched shape such as that in Fig. 4 is too complex for its straightforward application.
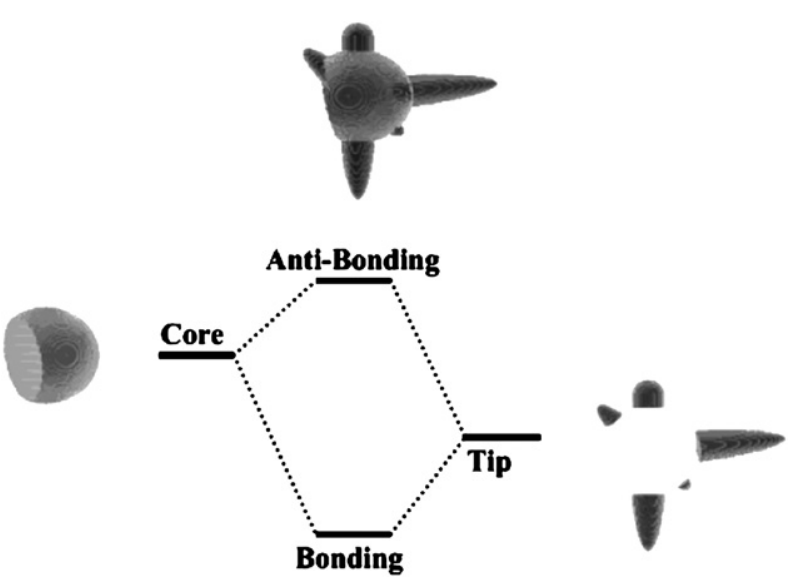

Fig. 5 The plasmon hybridization picture of gold nanostar LSPR. Adapted from reference 79 . 
However, using FDTD, the PH concept can be demonstrated by simulating the simpler structural subunits of the branched nanoparticles. The nanostar in Fig. 4 was therefore broken down into the spherical core and the elongated tips, and the LSPR of each was found by FDTD. ${ }^{79}$ Fig. 5 shows that the resulting LSPR energies can be thought of as a hybridization of the core and tip plasmons. Beyond simply setting the LSPR wavelength, this hybridization greatly increases the overall excitation cross section and field enhancements for the nanostar tips. This antenna effect of the nanostar core may be responsible for the relatively bright and narrow scattering spectra of nanostars in the single particle measurements. ${ }^{72}$

\section{Conclusions}

Advances in the synthesis of high yield, monodisperse gold and silver colloids of a variety of shapes have driven a new wave of interest in their optical properties and applications. These synthesis reactions are usually quite complex and not completely understood, but clearly sufficient for the progress to date. However, here we have shown that the LSPR of nanoparticles can be strongly affected by subtle aspects of their shape, and that more detailed properties can be found in more complicated geometries. Further advances in the field will therefore require further advances in our understanding of the nanoparticle synthesis and the chemical methods to assemble complex geometries.

\section{Acknowledgements}

This material is based upon work supported by, or in part by, the U. S. Army Research Laboratory and the U. S. Army Research Office under contract/grant number W911NF-04-1-0203. The authors also acknowledge support from the Welch Foundation under grant number C-1556.

\section{References}

1 S. Link and M. A. El-Sayed, J. Phys. Chem. B, 1999, 103, 4212-4217.

2 P. B. Johnson and R. W. Christy, Phys. Rev. B, 1972, 6, 4370-4379.

3 S. J. Oldenburg, R. D. Averitt, S. L. Westcott and N. J. Halas, Chem. Phys. Lett., 1998, 288, 243-247.

4 J. Y. Chen, B. Wiley, Z. Y. Li, D. Campbell, F. Saeki, H. Cang, L. Au, J. Lee, X. D. Li and Y. N. Xia, Adv. Mater., 2005, 17, 2255-2261.

5 A. Sanchez-Iglesias, I. Pastoriza-Santos, J. Perez-Juste, B. RodriguezGonzalez, F. J. G. de Abajo and L. M. Liz-Marzan, Adv. Mater., 2006, 18, 2529 .

6 M. Z. Liu and P. Guyot-Sionnest, J. Phys. Chem. B, 2005, 109, 22192-22200.

7 T. K. Sau and C. J. Murphy, Langmuir, 2004, 20, 6414-6420.

8 Y.-Y. Yu, S.-S. Chang, C.-L. Lee and C. R. C. Wang, J. Phys. Chem. $B, 1997,101,6661-6664$.

9 C. J. Johnson, E. Dujardin, S. A. Davis, C. J. Murphy and S. Mann, J. Mater. Chem., 2002, 12, 1765-1770.

10 N. R. Jana, L. Gearheart and C. J. Murphy, Adv. Mater., 2001, 13, 1389-1393.

11 B. Nikoobakht and M. A. El-Sayed, Chem. Mater., 2003, 15, 1957 1962.

12 T. K. Sau and C. J. Murphy, J. Am. Chem. Soc., 2004, 126, 86488649.

13 M. Grzelczak, J. Perez-Juste, F. J. G. de Abajo and L. M. Liz-Marzan, J. Phys. Chem. C, 2007, 111, 6183-6188.

14 J. W. Hotchkiss, A. B. Lowe and S. G. Boyes, Chem. Mater., 2007, 19, $6-13$.
15 X. S. Kou, S. Z. Zhang, Z. Yang, C. K. Tsung, G. D. Stucky, L. D. Sun, J. F. Wang and C. H. Yan, J. Am. Chem. Soc., 2007, 129, 6402 .

16 K. Mitamura, T. Imae, N. Saito and O. Takai, J. Phys. Chem. B, 2007, 111, 8891-8898.

17 Y. Niidome, K. Honda, K. Higashimoto, H. Kawazumi, S. Yamada, N. Nakashima, Y. Sasaki, Y. Ishida and J. Kikuchi, Chem. Commun., 2007, 3777-3779.

18 R. T. Tom, A. K. Samal, T. S. Sreeprasad and T. Pradeep, Langmuir, 2007, 23, 1320-1325.

19 M. Grzelczak, J. Perez-Juste, B. Rodriguez-Gonzalez and L. M. Liz-Marzan, J. Mater. Chem., 2006, 16, 3946-3951.

20 M. Z. Liu and P. Guyot-Sionnest, J. Mater. Chem., 2006, 16, 39423945.

21 I. Pastoriza-Santos, J. Perez-Juste and L. M. Liz-Marzan, Chem. Mater., 2006, 18, 2465-2467.

22 H. Takahashi, Y. Niidome, T. Niidome, K. Kaneko, H. Kawasaki and S. Yamada, Langmuir, 2006, 22, 2-5.

23 C. K. Tsung, X. S. Kou, Q. H. Shi, J. P. Zhang, M. H. Yeung, J. F. Wang and G. D. Stucky, J. Am. Chem. Soc., 2006, 128, 53525353.

24 M. Jebb, P. K. Sudeep, P. Pramod, K. G. Thomas and P. V. Kamat, J. Phys. Chem. B, 2007, 111, 6839-6844.

25 H. Nakashima, K. Furukawa, Y. Kashimura and K. Torimitsu, Chem. Commun., 2007, 1080-1082.

26 B. F. Pan, D. X. Cui, C. G. Ozkan, P. Xu, T. Huang, Q. Li, H. Chen, F. T. Liu, F. Gao and R. He, J. Phys. Chem. C, 2007, 111, 1257212576.

27 S. Pierrat, I. Zins, A. Breivogel and C. Sonnichsen, Nano Lett., 2007, 7, 259-263.

28 M. Spuch-Calvar, J. Perez-Juste and L. M. Liz-Marzan, J. Colloid Interface Sci., 2007, 310, 297-301.

29 S. Vial, I. Pastoriza-Santos, J. Perez-Juste and L. M. Liz-Marzan, Langmuir, 2007, 23, 4606-4611.

30 M. H. Zareie, X. D. Xu and M. B. Cortie, Small, 2007, 3, 139-145.

31 S. T. S. Joseph, B. I. Ipe, P. Pramod and K. G. Thomas, J. Phys. Chem. B, 2006, 110, 150-157.

32 M. Pelton, M. Z. Liu, H. Y. Kim, G. Smith, P. Guyot-Sionnest and N. E. Scherer, Opt. Lett., 2006, 31, 2075-2077.

33 J. Aizpurua, G. W. Bryant, L. J. Richter, F. J. G. de Abajo, B. K. Kelley and T. Mallouk, Phys. Rev. B, 2005, 71, 235420.

34 C. D. Chen, S. F. Cheng, L. K. Chau and C. R. C. Wang, Biosens. Bioelectron., 2007, 22, 926-932.

35 M. Das, N. Sanson, D. Fava and E. Kumacheva, Langmuir, 2007, 23, 196-201.

36 H. Ding, K. T. Yong, I. Roy, H. E. Pudavar, W. C. Law, E. J. Bergey and P. N. Prasad, J. Phys. Chem. C, 2007, 111, 12552-12557.

37 N. J. Durr, T. Larson, D. K. Smith, B. A. Korgel, K. Sokolov and A. Ben-Yakar, Nano Lett., 2007, 7, 941-945.

38 M. Eghtedari, A. Oraevsky, J. A. Copland, N. A. Kotov, A. Conjusteau and M. Motamedi, Nano Lett., 2007, 7, 1914-1918.

39 Y. Horiguchi, T. Niidome, S. Yamada, N. Nakashima and Y. Niidome, Chem. Lett., 2007, 36, 952-953.

40 X. H. Huang, I. H. El-Sayed, W. Qian and M. A. El-Sayed, Nano Lett., 2007, 7, 1591-1597.

41 T. B. Huff, M. N. Hansen, Y. Zhao, J. X. Cheng and A. Wei, Langmuir, 2007, 23, 1596-1599.

42 V. R. R. Kumar, A. K. Samal, T. S. Sreeprasad and T. Pradeep, Langmuir, 2007, 23, 8667-8669.

43 S. M. Marinakos, S. H. Chen and A. Chilkoti, Anal. Chem., 2007, 79, $5278-5283$.

44 A. K. Olyelere, P. C. Chen, X. Huang, I. H. El-Sayed and M. A. El-Sayed, Bioconjugate Chem., 2007, 18, 1490-1497.

45 A. Shiotani, T. Mori, T. Niidome, Y. Niidome and Y. Katayama, Langmuir, 2007, 23, 4012-4018.

46 J. W. Stone, P. N. Sisco, E. C. Goldsmith, S. C. Baxter and C. J. Murphy, Nano Lett., 2007, 7, 116-119.

47 C. X. Yu and J. Irudayaraj, Anal. Chem., 2007, 79, 572-579.

48 C. X. Yu, H. Nakshatri and J. Irudayaraj, Nano Lett., 2007, 7, 2300 2306.

49 X. Huang, I. H. El-Sayed, W. Qian and M. A. El-Sayed, J. Am. Chem. Soc., 2006, 128, 2115-2120.

50 M. Rex, F. E. Hernandez and A. D. Carnpiglia, Anal. Chem., 2006, 78, 445-451. 
51 C. C. Chen, Y. P. Lin, C. W. Wang, H. C. Tzeng, C. H. Wu, Y. C. Chen, C. P. Chen, L. C. Chen and Y. C. Wu, J. Am. Chem. Soc., 2006, 128, 3709-3715.

52 C. J. Orendorff, L. Gearheart, N. R. Jana and C. J. Murphy, Phys. Chem. Chem. Phys., 2006, 8, 165-170.

53 C. X. Yu, L. Varghese and J. Irudayaraj, Langmuir, 2007, 23, 9114 9119.

54 C. Noguez, J. Phys. Chem. C, 2007, 111, 3806-3819.

55 I. Pastoriza-Santos, A. Sanchez-Iglesias, F. J. G. de Abajo and L. M. Liz-Marzan, Adv. Funct. Mater., 2007, 17, 1443-1450.

56 D. P. Sprunken, H. Omi, K. Furukawa, H. Nakashima, I. Sychugov, Y. Kobayashi and K. Torimitsu, J. Phys. Chem. C, 2007, 111, 14299 14306.

57 S. Link, M. Mohamed and M. El-Sayed, J. Phys. Chem. B, 1999, 103, 3073-3077.

58 H. Liao and J. Hafner, Chem. Mater., 2005, 17, 4636-4641.

59 C. J. Orendorff and C. J. Murphy, J. Phys. Chem. B, 2006, 110, 3990 3994.

60 C. Foss, G. Hornyak, J. Stockert and C. Martin, J. Phys. Chem., 1994, 98, 2963-2971.

61 E. K. Payne, K. L. Shuford, S. Park, G. C. Schatz and C. A. Mirkin, J. Phys. Chem. B, 2006, 110, 2150-2154.

62 A. Brioude, X. C. Jiang and M. P. Pileni, J. Phys. Chem. B, 2005, 109, $13138-13142$.

63 B. N. Khlebtsov and N. G. Khlebtsov, J. Phys. Chem. C, 2007, 111, $11516-11527$.

64 X. D. Xu and M. B. Cortie, Adv. Funct. Mater., 2006, 16, 2170-2176.

65 S. Chen, Z. Wang, J. Ballato, S. Foulger and D. Carroll, J. Am. Chem. Soc., 2003, 125, 16186-16187.

66 E. Hao, R. Bailey, G. Schatz, J. Hupp and S. Li, Nano Lett., 2004, 4, $327-330$.
67 M. Yamamoto, Y. Kahiwagi, T. Sakata, H. Mori and M. Nakamoto, Chem. Mater., 2005, 17, 5391-5393.

68 C. H. Kuo and M. H. Huang, Langmuir, 2005, 21, 2012-2016.

69 J. L. Burt, J. L. Elechiguerra, J. Reyes-Gasga, J. M. MontejanoCarrizales and M. Jose-Yacaman, J. Cryst. Growth, 2005, 285, 681691

70 M. Zhou, S. H. Chen and S. Y. Zhao, Chem. Lett., 2006, 35, 332333.

71 H. Y. Wu, M. Liu and M. H. Huang, J. Phys. Chem. B, 2006, 110, 19291-19294.

72 C. L. Nehl, H. W. Liao and J. H. Hafner, Nano Lett., 2006, 6, 683688.

73 L. Lu, A. Kobayashi, K. Tawa and Y. Ozaki, Chem. Mater., 2006, 18, 4894-4901.

74 O. M. Bakr, B. H. Wunsch and F. Stellacci, Chem. Mater., 2006, 18, 3297-3301.

75 H. Yuan, W. H. Ma, C. C. Chen, J. C. Zhao, J. W. Liu, H. Y. Zhu and X. P. Gao, Chem. Mater., 2007, 19, 1592-1600.

76 J. P. Xie, J. Y. Lee and D. I. C. Wang, Chem. Mater., 2007, 19, $2823-$ 2830.

77 Y. H. Su, W. H. Lai, W. Y. Chen, M. H. Hon and S. H. Chang, Appl. Phys. Lett., 2007, 90, 181905.

78 O. Krichevski and G. Markovich, Langmuir, 2007, 23, 1496-1499.

79 F. Hao, C. L. Nehl, J. H. Hafner and P. Nordlander, Nano Lett., 2007, 7, 729-732.

80 C. Oubre and P. Nordlander, J. Phys. Chem. B, 2005, 109, 1004210051.

81 E. Prodan, C. Radloff, N. J. Halas and P. Nordlander, Science, 2003 , 302, 419-422.

82 H. Wang, D. W. Brandl, P. Nordlander and N. J. Halas, Acc. Chem. Res., 2007, 40, 53-62. 